\title{
EFECTIVIDAD EN LA SELECCIÓN DE BENEFICIARIOS DE LOS PROGRAMAS AVANCEMOS Y BIENESTAR FAMILIAR ${ }^{1}$
}

\section{EFFECTIVENESS IN THE SELECTION OF BENEFICIARIES OF THE AVANCEMOS AND BIENESTAR FAMILIAR PROGRAMS}

\section{Francisco Delgado Jiménez ${ }^{2}$}

\begin{abstract}
Resumen
Este análisis se centra en dos programas sociales gestionados por el Instituto Mixto de Ayuda Social (IMAS) para combatir la pobreza: Avancemos y Bienestar Familiar. El objetivo principal de este estudio es identificar la efectividad en la asignación de los beneficios de dichos programas, con el fin de corregir los mecanismos que a) han permitido a los no pobres obtener beneficios $\mathrm{y}$ b) han impedido que los hogares elegibles accedan a los beneficios. Datos de la Encuesta Nacional de Hogares señalan importantes filtraciones y además una cobertura limitada de la población objetivo, que ponen en evidencia la necesidad de ajustar los mecanismos de focalización, de forma tal que la inversión social pública destinada a programas selectivos tenga incidencia en la reducción de la pobreza en Costa Rica.
\end{abstract}

Palabras clave: protección social; programas sociales; bienestar social; pobreza; focalización

Doi: http://dx.doi.org/10.15359/eys.22-52.1

Fecha de recepción: 12-12-2016. Fechas de reenvíos: 19-12-2016, 02-02-2017, 13-02-2017, 09-03-2017, 07-042017, 10-05-2017. Aceptado el 09-06-2017. Publicado el 01-07-2017.

${ }^{1}$ Este trabajo es una adaptación del proyecto de graduación para optar por el grado de Máster en Administración Pública en Desarrollo Internacional de la Escuela de Gobierno John F. Kennedy de la Universidad de Harvard, con datos actualizados para los años 2015 y 2016.

${ }^{2}$ Licenciado en Economía y Máster en Desarrollo Internacional. Economista de Políticas Sociales, Instituto Mixto de Ayuda Social (IMAS), Costa Rica. Académico, Escuela de Economía, Universidad de Costa Rica, Costa Rica. Correo electrónico francisco.delgado@ucr.ac.cr

Francisco Delgado Jiménez

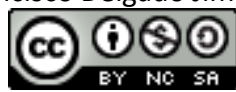

Revista Economía y Sociedad by Universidad Nacional is licensed under a CreativeCommons Reconocimiento-NoComercial- 


\begin{abstract}
This analysis focuses on two social programs managed by the Joint Institute for Social Assistance (IMAS) in order to reduce poverty: Avancemos and Bienestar Familiar. The main objective of this study is to identify the effectiveness in allocating benefits of such programs, in order to correct the mechanisms that have a) allowed non-poor individuals receive benefits and b) prevented eligible households to access the benefits. Data from the National Household Survey points out important leakages and a limited coverage of the target population, which highlights the need to adjust targeting mechanisms, so that the public social investment targeted to selected programs has an impact on reducing poverty in Costa Rica.
\end{abstract}

Keywords: social protection; social programs; social welfare; poverty; targeting

\title{
Introducción
}

La asistencia social juega un papel clave en un conjunto multidimensional de políticas que los gobiernos pueden implementar para combatir la pobreza. Los servicios universales y las políticas de mercado de trabajo son también parte de ese conjunto de herramientas que coexisten con las transferencias monetarias y en especie dirigidas a poblaciones específicas. Una característica central de estas transferencias es su enfoque de corto plazo para la reducción de la pobreza, ya que ayuda a resolver el problema de la pobreza en la medida en que se otorguen los beneficios, por lo que los gobiernos deben poner especial atención a la selección de los beneficiarios. Las restricciones fiscales exigen eficiencia en la asignación de esas transferencias, pero esta distribución eficiente es también una cuestión de justicia social, ya que genera oportunidades para fomentar el desarrollo y la inclusión.

De acuerdo con Coady, Grosh and Hoddinott (2004), "la focalización es un medio para aumentar la eficiencia del programa, aumentando los beneficios que los pobres pueden obtener dentro de un programa con presupuesto fijo" (p. 5). En este sentido, un programa social focalizado, con grupos específicos que obtienen ciertos beneficios difiere de un programa universal dirigido a toda la población. En las sociedades donde la pobreza y la desigualdad de ingresos son asuntos relevantes, la focalización se convierte en una herramienta importante para dirigir la asistencia social a los que no tienen las capacidades y oportunidades para mejorar su bienestar.

Un supuesto detrás de este enfoque consiste en que es posible distinguir quién es pobre y quién no. En algunos casos, la información disponible de los hogares permite una identificación más fácil de sus condiciones de vida; sin embargo, en países en desarrollo podrían ni siquiera existir datos, teniendo que depender de herramientas para aproximar los niveles de vida de los

2

Francisco Delgado Jiménez

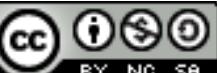

Revista Economía y Sociedad by Universidad Nacional is licensed under a CreativeCommons Reconocimiento-NoComercial- 
hogares. Como se ha mencionado, este trabajo se centra en dos programas sociales gestionados por el Instituto Mixto de Ayuda Social (IMAS): Avancemos y Bienestar Familiar.

El programa Avancemos ofrece un auxilio económico para sustituir las remuneraciones que recibirían los jóvenes en el trabajo, buscando con ello reducir la deserción escolar mediante el aumento del ingreso disponible de los hogares. Se han observado resultados positivos a corto plazo y también se espera un impacto positivo de este programa a largo plazo. Aunque Avancemos tiene un impacto positivo en la asistencia escolar, 1 de cada 4 estudiantes que se beneficiaron del programa de becas en 2014 pertenecía a un hogar que no era ni pobre ni vulnerable a la pobreza, y alrededor del $60 \%$ de estudiantes de hogares pobres y de ingresos bajos no estaban recibiendo el beneficio. Por otra parte, la adecuada selección de beneficiarios es de especial interés dado que la mayor parte del gasto social se dirige a los programas universales y menos del $15 \%$ se dedica a programas selectivos.

Por otra parte, Bienestar Familiar ofrece una transferencia para las familias que viven en condiciones de pobreza para cubrir diferentes necesidades o atender ciertas situaciones coyunturales (por ejemplo, transferencias para cubrir alimentación y servicios básicos, educación y formación, cuido de niños y gastos en salud). Sin embargo, el IMAS ha tenido dificultades para aumentar la cobertura de este programa más allá del $10 \%$ de toda la población objetivo, no solo por falta de recursos sino también porque casi el $40 \%$ de los beneficios se asignan a hogares que no son pobres.

Los principales objetivos de este estudio son examinar el proceso de selección de los beneficiarios de los programas sociales y ofrecer recomendaciones para corregir los canales y mecanismos que, intencionalmente o no, han permitido a los no pobres beneficiarse de ellos y han excluido a los más necesitados.

La primera sección resume la evolución de la pobreza y la inversión social pública en Costa Rica, identificando el rol central del IMAS en el combate a la pobreza. Esta sección también contiene una descripción de los dos programas focalizados y el mecanismo de selección que este trabajo indaga. Luego, se explican aspectos de orden metodológico, específicamente lo relacionado con definiciones de pobreza y el uso de las bases de datos de la Encuesta de Hogares. Seguidamente, se analiza el desempeño de la focalización del sistema actual, tomando en cuenta diferentes enfoques de medición de la pobreza. Se describen los principales inconvenientes de la situación actual y se detallan algunas recomendaciones. Por último, se incluyen algunas consideraciones finales. 


\section{Pobreza y programas sociales en Costa Rica}

\subsection{Pobreza en Costa Rica}

A partir de la crisis económica que afectó Costa Rica en los años 80, la mayoría de los indicadores sociales y económicos se deterioraron drásticamente. No fue sino hasta la siguiente década que algunos de ellos comenzaron a recuperar su trayectoria anterior. La pobreza mostró una importante disminución entre 1992 y 1994, pero desde entonces y a pesar de un crecimiento económico estable ${ }^{3}$ y un aumento en los recursos asignados a los programas sociales (Figura 2), la tasa de pobreza se ha mantenido en torno al $20 \%$ y la pobreza extrema ha afectado en promedio casi al $6 \%$ de los hogares del país (ver Figura 1).

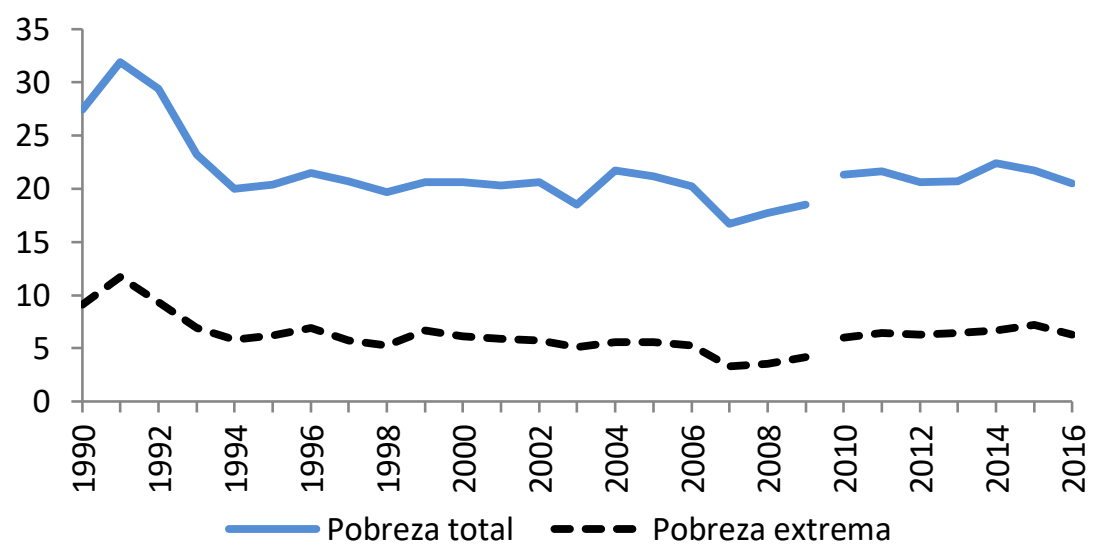

Figura 1. Incidencia de pobreza y pobreza extrema en Costa Rica 1990-2014. Nota: Cambio metodológico en 2010. Fuente: elaboración propia con datos del Instituto Nacional de Estadística y Censos (INEC).

Investigaciones e informes anteriores han tratado de explicar este fenómeno, destacando varias causas. Por ejemplo, la Contraloría General de la República (CGR, 2006) sostiene que los programas sociales en Costa Rica son de naturaleza diversa, repartidos entre un gran número de instituciones y no constituyen una política integrada o cohesiva, lo que reduce la capacidad de luchar contra la pobreza. Sauma y Trejos (2014) muestran que algunos programas sociales presentan filtraciones importantes, y Trejos y Sáenz (2007) destacan el hecho de que no se ha desarrollado completamente un registro uniforme de los beneficiarios y de la población objetivo.

\footnotetext{
3 En promedio, el crecimiento real anual durante el período 1994-2016 fue de 4.5\%. Las tasas de desempleo tuvieron un comportamiento estable durante ese período (controlando por cambios metodológicos), aunque ha mostrado una tendencia creciente desde de la crisis de 2009.
}

4

Francisco Delgado Jiménez

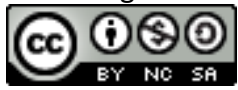

Revista Economía y Sociedad by Universidad Nacional is licensed under a CreativeCommons Reconocimiento-NoComercial- 


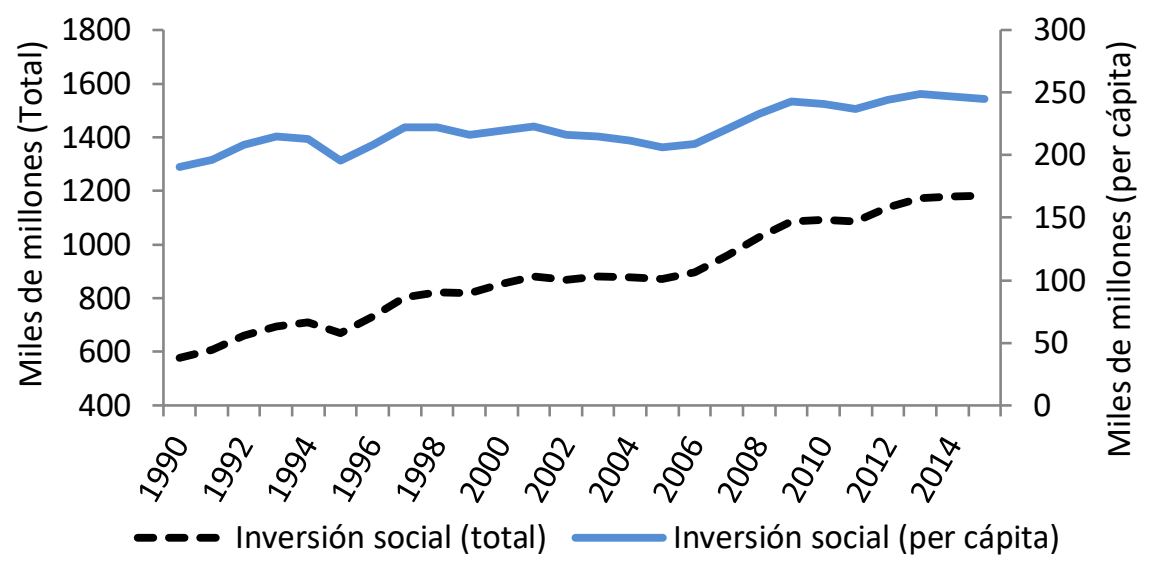

Figura 2. Evolución de la Inversión Pública Social en Costa Rica 1990-2013 1/. Notas: 1/ En términos reales. Colones del año 2000, deflactado con el índice de precios implícito del gasto de consumo del Gobierno Central. Fuente: elaboración propia con base en datos de la Secretaría Técnica de la Autoridad Presupuestaria (STAP).

\subsection{El rol del Instituto Mixto de Ayuda Social}

La creación del IMAS en 1971 cambió en gran medida la estructura de la asistencia social y la prestación de servicios a las familias necesitadas. Esta mejora institucional significó la consolidación de los programas sociales focalizados que vino a complementar la larga trayectoria de prestación universal de servicios públicos en Costa Rica. Es posible inferir esta visión a partir de la exposición de motivos en el proyecto de ley que fue objeto de debate en el Congreso en ese momento:

Es tarea del Estado distribuir, de la manera más equitativa, la riqueza nacional. El ayudar a quien hoy vive en miseria extrema no debe conceptuarse como una carga o un impedimento al desarrollo integral de la nación. Todo lo contrario: la inversión que el Estado realiza para ofrecerle un mínimo standard de vida a este sector de la población es tan reproductiva como la inversión en salud o educación. (Asamblea Legislativa, $\underline{1970}$, p. 2)

Solís (2012) explica que durante las reformas económicas de la década de 1990 y principios de 2000 el IMAS pasó por un proceso de modernización a través de la introducción de Sistemas de Información Social y una reorientación de los programas ofrecidos a los pobres para incentivar el desarrollo de destrezas y talento humano. A partir de la década de 1990, el IMAS creó un Sistema de Selección de Beneficiarios (SISBEN), una adaptación del esquema chileno CAS, que fue el primer intento de utilizar criterios técnicos para focalizar el gasto social. Más tarde, entre 2000 y 2002, el IMAS y el Programa de las Naciones Unidas para el Desarrollo (PNUD) actualizaron ese instrumento y crearon el Sistema de Información de Población Objetivo (SIPO).

Francisco Delgado Jiménez

(c) (†) 8 ()

Revista Economía y Sociedad by Universidad Nacional is licensed under a CreativeCommons Reconocimiento-NoComercial- 
EI SIPO, con algunas mejoras en su metodología y alcance, es actualmente el instrumento que establece los criterios para la selección de beneficiarios.

\subsection{Principales programas focalizados: Avancemos y Bienestar Familiar}

- Población objetivo y prioridades

El IMAS es una de las principales instituciones en términos de protección social y es parte de la base del Estado de bienestar en Costa Rica. Para el combate a la pobreza, está encargada de la administración de varios programas con diferentes propósitos, entre ellos: Avancemos y Bienestar Familiar.

Avancemos se introdujo en el año 2006 y ofrece una transferencia monetaria condicionada a las familias con jóvenes entre 12 y 25 años de edad, que viven en condiciones de pobreza o vulnerabilidad social y requieren de un incentivo económico para permanecer en el sistema educativo secundario en vez de ingresar al mercado laboral. El objetivo principal de Avancemos es mantener a los estudiantes en el sistema escolar compensando el ingreso potencial obtenido en el trabajo al abandonar el colegio. Se inició en 2006 como un plan piloto, proporcionando becas a 8.000 estudiantes $y$, desde entonces, ha aumentado su cobertura, como se observa en la Tabla $\underline{1}$.

Tabla 1.

Evolución de beneficiarios de Avancemos y Bienestar Familiar

\begin{tabular}{rrrr}
\hline & \multicolumn{2}{c}{ Avancemos } & Bienestar Familiar \\
(Estudiantes) & \multicolumn{1}{c}{ (Familias) } & \multicolumn{1}{c}{ (Familias) } \\
\hline 2006 & 8.137 & 6.988 & 23.656 \\
2007 & 52.419 & 39.872 & 24.437 \\
2008 & 55.687 & 41.398 & 41.166 \\
2009 & 165.749 & 129.091 & 57.036 \\
2010 & 185.214 & 138.415 & 57.081 \\
2011 & 185.315 & 139.665 & 67.285 \\
2012 & 181.570 & 137.563 & 78.367 \\
2013 & 171.534 & 133.149 & 84.409 \\
2014 & 174.196 & 136.214 & 83.959 \\
2015 & 171.203 & 133.357 & 118.998 \\
\hline
\end{tabular}

Fuente: elaboración propia con datos del Sistema de Atención a Beneficiarios del IMAS.

El otro programa en estudio, llamado Bienestar Familiar, responde al objetivo central del IMAS de "luchar contra la pobreza extrema". Esta transferencia de efectivo no condicionada está orientada a aumentar el ingreso disponible de las familias y hacerlos capaces de cubrir ciertas necesidades.

6

Francisco Delgado Jiménez

(c) (†) ()

Revista Economía y Sociedad by Universidad Nacional is licensed under a CreativeCommons Reconocimiento-NoComercial- 
Sistema de Información de Población Objetivo (SIPO) es el mecanismo que se utiliza para generar un puntaje para cada familia, y se utiliza en la actualidad como el criterio técnico para seleccionar a los beneficiarios de Avancemos y Bienestar Familiar.

De acuerdo con los manuales y procedimientos para la selección de los beneficiarios del IMAS, después de que una familia o individuo aplica para un programa social en cualquiera de las oficinas del IMAS ${ }^{4}$, un trabajador social visita el hogar y completa la Ficha de Información Social (FIS). Esta herramienta proporciona información útil sobre las condiciones de vida de las familias: las instalaciones de la vivienda, los servicios básicos, la información de empleo, fuentes de ingresos, el acceso a la seguridad social, el nivel educativo y la propiedad de varios artefactos. La información se utiliza como insumo para calcular el "puntaje SIPO", que es básicamente un "proxy means test" ${ }^{\text {. }}$. Basado en un modelo econométrico con 16 variables, la información se transforma en un puntaje para cada familia que determina si finalmente es elegible o no para recibir beneficios.

Dada la constitución metodológica estadística del instrumento y su amplio rango de variación, el SIPO establece un riguroso ordenamiento de los postulantes, donde el menor puntaje se asocia a una pobreza mayor y más intensa. La idea del puntaje es asegurar con objetividad y equidad que los postulantes tendrán igualdad de oportunidades para acceder a un beneficio según su condición de carencia. (Víquez, $\underline{2005}$, p. 15)

EI IMAS desarrolló una clasificación de las familias con el fin de dar prioridad a la asistencia social. De acuerdo con el puntaje SIPO, las familias se agrupan en cuatro categorías. Aquellos en el grupo 1 son los más pobres y tienen mayor prioridad; el grupo 2 representa la pobreza moderada y ocupa el segundo lugar en prioridad. El grupo 3 corresponde a las familias en condiciones vulnerables, bajo nivel de vida y propensos a la pobreza. Por último, el grupo 4 incluye las familias que no son una prioridad para recibir atención social. En el Anexo 1, se

\footnotetext{
${ }^{4}$ Actualmente, los programas gestionados por el IMAS se ejecutan en su mayoría "por demanda", lo que significa que casi la totalidad de los beneficiarios se auto-identifican como posibles candidatos y aplican para esos programas. En el caso de la Estrategia Puente al Desarrollo, un nuevo modelo de atención introducido en 2015, la figura de la persona cogestora social cambia ese paradigma, ya que constituye una forma en que la institución hace una búsqueda activa de potenciales beneficiarios.

${ }^{5}$ Según lo explicado por Coady, Grosh y Hoddinott (2004), en los países en desarrollo, la falta de información disponible sobre el ingreso y el patrimonio hace muy difícil evaluar si un solicitante es elegible para un programa o no. La segunda mejor opción es un proxy means test, que es una aproximación de las situaciones socioeconómicas, mediante verificación parcial y entrevistas. Este método consiste en un sistema que genera una puntuación para los hogares candidatos; el verdadero reto es crear partituras que interpretan lo más fielmente posible la realidad socioeconómica de cada hogar en relación con la realidad de la población media, con el fin de priorizar adecuadamente los hogares y para garantizar el acceso equitativo a los beneficios sociales.
}

Francisco Delgado Jiménez

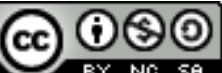

Revista Economía y Sociedad by Universidad Nacional is licensed under a CreativeCommons Reconocimiento-NoComercial- 
describe cómo funciona este modelo de puntaje. En línea con su objetivo clave para combatir la pobreza extrema, el IMAS se supone que debe proporcionar beneficios en ese orden, pero no siempre ha sido el caso.

Recientemente, la Presidencia Ejecutiva emitió una nueva directriz para extender los beneficios a las familias de los grupos 1 y 2 solamente. Excepcionalmente, y solo mediante la evaluación de un trabajador social o en el caso de los hogares encabezados por mujeres, invalidez 0 enfermedad terminal de un miembro del hogar, el IMAS puede proporcionar beneficios a las personas o familias en el grupo 3, siempre que no superen la línea de pobreza (IMAS, 2014). Este nuevo criterio se aplica tanto para Avancemos como para Bienestar Familiar. Los resultados de esta medida se comentan en el acápite 3.1.

Cuando una familia es seleccionada para recibir los beneficios, el período en que permanecen como beneficiarios varía entre Avancemos y Bienestar Familiar. En el caso del programa de becas, hay una evaluación cada un año y medio. Si las condiciones socioeconómicas de las familias no han cambiado, siguen recibiendo los beneficios, siempre y cuando cumplan también la condición de asistencia al colegio. Para Bienestar Familiar, la duración del programa varía dependiendo de la razón que motivó la transferencia originalmente.

\section{Aspectos metodológicos}

La focalización de estos programas se realiza utilizando proxy means tests, la segunda mejor opción, por lo que es necesario considerar los errores de inclusión y exclusión. Un error de inclusión o de filtración se produce cuando un hogar no pobre está incorrectamente identificado como pobre y, por lo tanto, se inscriben en el programa, aunque en realidad no deberían ser elegibles. Por otra parte, un error de exclusión se produce cuando el sistema identifica a las personas pobres como no pobres y les niega el acceso al programa (Coady, Grosh, Hoddinott, 2004).

Un enfoque común para evaluar el desempeño de la focalización es comparar las tasas de subcobertura y de filtración. La tasa de subcobertura es la proporción de hogares pobres que no están incluidos en el programa (error de exclusión) y la tasa de filtración es la proporción de los no pobres que son incluidos en el programa (error de inclusión).

$$
\begin{aligned}
\text { Filtración } & =\operatorname{Pr}(\text { no pobre } \mid \text { incluido en programa }) \\
& =\frac{\operatorname{Pr}(\text { no pobre e incluido en programa })}{\operatorname{Pr}(\text { incluido en programa })}
\end{aligned}
$$

Subcobertura $=\operatorname{Pr}($ no incluido en programa $\mid$ pobre $)$

$$
=\frac{\operatorname{Pr}(\text { no incluido en programa y pobre })}{\operatorname{Pr}(\text { pobre })}
$$

8 
Estos indicadores se estiman para ambos programas tomando en cuenta el método de Línea de Pobreza ${ }^{6}$, con el fin de evidenciar la capacidad de los sistemas del IMAS de distinguir entre hogares pobres y no pobres. Para este ejercicio, se utilizan los datos de la Encuesta Nacional de Hogares (ENAHO) de 2014 (Instituto Nacional de Estadística y Censos, 2014).

Estos mismos datos permiten realizar una simulación del modelo econométrico que utiliza el IMAS para escoger a los beneficiarios de los programas (SIPO). Se realizará un ejercicio donde a cada uno de los hogares contenidos en la encuesta se les asigna un puntaje como si estuvieran aplicando para un programa del IMAS. Esto posibilita identificar la distribución que genera el modelo econométrico, así como la comparación del puntaje con el método de línea de pobreza.

\section{Estado actual de la focalización de los programas}

Como primer paso, resulta útil analizar la distribución de los beneficiarios de los programas por puntaje, como se observa en la Figura $\underline{3}$. De acuerdo con los registros del IMAS, el 94,5\% de los beneficiarios de Bienestar Familiar en 2014 fueron clasificados en los grupos 1 y 2 (sujeto de asistencia social), lo que significa que al parecer el mecanismo de selección es tan eficiente que solo permitía un índice de filtraciones de 5,5\%. En el caso de Avancemos, la tasa de filtraciones observada es del $24,8 \%$, probablemente asociada al tratamiento de estas becas como un programa masivo. En todo caso, las tasas de filtración observadas no parecen altas, a primera vista.

A continuación, se realiza un ejercicio con mayor detalle, tomando como base la información contenida en la ENAHO 2014, en lugar de los registros oficiales del IMAS. Como se comenta más adelante, una de las inconsistencias entre el modelo de atención del IMAS y las cifras oficiales de pobreza radica en que la utilización de unidades de análisis distintas: el IMAS utiliza la familia como criterio, mientras que INEC parte de una definición de hogar ${ }^{7}$.

\footnotetext{
${ }^{6}$ El método de línea de pobreza es la determinación de la condición de pobreza comparando el ingreso per cápita del hogar con el costo de una canasta de bienes y servicios básicos.

${ }^{7}$ De acuerdo con el Manual para la Aplicación de la Ficha de Información Social del IMAS (Instituto Mixto de Ayuda Social, 2009), la familia se define con base en lazos de consanguinidad, mientras que en el caso de la ENAHO, el hogar consiste en relaciones económicas, donde se comparten alimentos y presupuesto entre personas que residen en una misma vivienda.
}

Francisco Delgado Jiménez

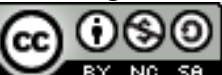

Revista Economía y Sociedad by Universidad Nacional is licensed under a CreativeCommons Reconocimiento-NoComercial- 


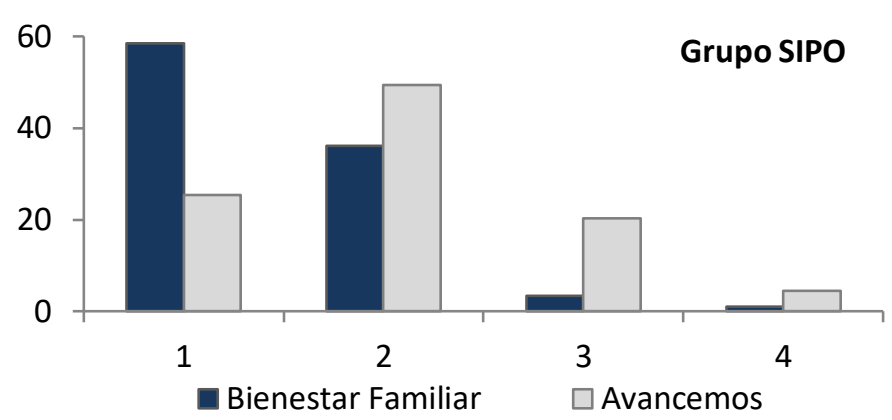

Figura 3. Distribución de beneficiarios por grupo de puntaje de acuerdo con registros del SIPO, 2014. Fuente: elaboración propia con datos del Sistema de Información de Población Objetivo del IMAS.

\subsection{Método de línea de pobreza}

Si la pobreza se define con base en el ingreso de los hogares (método de la línea de pobreza), la Encuesta Nacional de Hogares 2014 permite observar que el sistema del IMAS muestra grandes filtraciones y de subcobertura, lo que reducen la probabilidad de alcanzar los objetivos finales de los programas. La Tabla $\underline{2}$ resume los principales indicadores de desempeño de los programas de interés. El programa Avancemos cubre menos de la mitad de los beneficiarios potenciales (estudiantes de secundaria entre las edades de 12 y 25 en situación de pobreza o condiciones de vulnerabilidad) y muestra filtraciones relativamente altas.

Avancemos inició como un programa masivo con un objetivo secundario en mente: reducir la deserción en la educación secundaria, lo que puede explicar la gran proporción de beneficiarios por encima de la línea de pobreza. Si se consideran otros hogares vulnerables (primeros cuatro deciles), la medida de filtraciones llega al 33\%. El caso de Bienestar Familiar es diferente: tiene una tasa de cobertura aún más baja, pero está relativamente mejor focalizado si se incluye a la población vulnerable. Sin embargo, en este caso, el programa fue pensado para reducir la pobreza extrema y luego se expandió para todos los hogares pobres; según el mismo criterio, la tasa de filtraciones representa el $36,7 \%$ de los hogares. 
Tabla 2.

Desempeño de la focalización de Avancemos y Bienestar Familiar

\begin{tabular}{lcccc}
\hline \multirow{2}{*}{ Programa } & \multicolumn{2}{c}{ Subcobertura } & \multicolumn{2}{c}{ Filtraciones $^{5 /}$} \\
\cline { 2 - 5 } & $\begin{array}{c}\text { Línea de } \\
\text { pobreza }\end{array}$ & 40 menor $^{2 /}$ & $\begin{array}{c}\text { Línea de } \\
\text { pobreza }^{3 /}\end{array}$ & 40 menor $^{4 /}$ \\
\hline Avancemos (\% de estudiantes) & 56,3 & 57,9 & 58,9 & 33,0 \\
Bienestar Familiar (\% de familias) & 93,9 & 95,2 & 36,7 & 12,6 \\
\hline
\end{tabular}

Notas:

1/ Proporción de familias o estudiantes pobres no cubiertos por el programa.

2/ Proporción de hogares en los primeros cuatro deciles en la distribución del ingreso no cubiertos por el programa.

3/ Proporción de los beneficiaros de los programas por encima de la línea de pobreza.

4/ Proporción de beneficiarios de los programas en los últimos seis deciles de la distribución del ingreso. 5/ En el caso de Avancemos, las filtraciones también incluyen a aquellos que no cumplen con los requisitos del programa (edad o matrícula) pero que aun así reciben beneficios.

Fuente: elaboración propia con base en datos de INEC (2014).

A finales de 2014, la Presidencia Ejecutiva del IMAS emitió una Directriz de Priorización de Atención de la Pobreza para limitar las ayudas del IMAS a los grupos de puntaje 1 y 2 (Instituto Mixto de Ayuda Social, 2014). Esta medida tuvo resultados positivos: entre 2014 y 2015, en el programa Avancemos se observa una reducción mínima en las filtraciones, pero en el programa Bienestar Familiar se evidencia una reducción significativa de las filtraciones, con respecto al año anterior (Figura 4). Si se utiliza el método de línea de pobreza, las filtraciones se redujeron en 5,1 puntos porcentuales. Entre 2015 y 2016, la proporción de subcobertura y filtraciones prácticamente no cambia para ninguno de los dos programas.

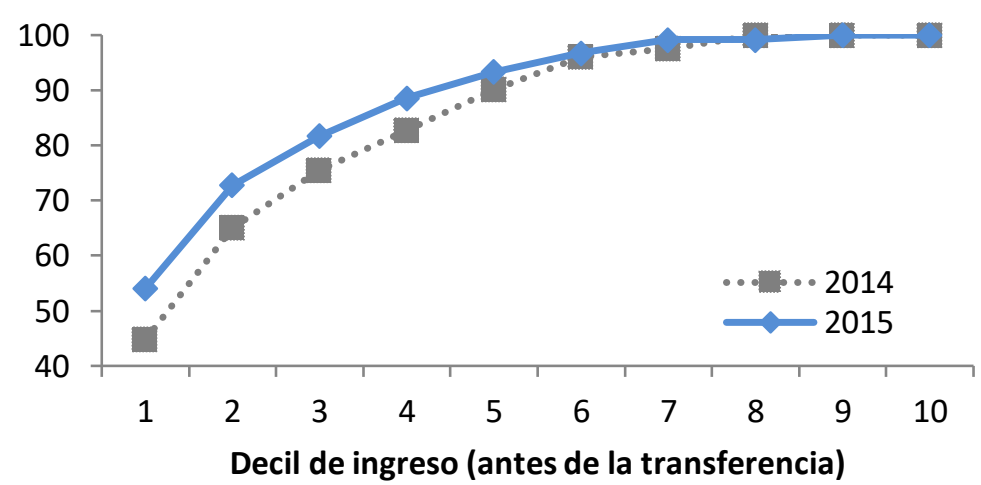

Figura 4. Distribución acumulada de los beneficios de Bienestar Familiar según decil de ingreso per cápita antes de la transferencia para los años 2014 y 2015. Fuente: elaboración propia con base en datos de INEC (2014). 


\subsection{Simulación del modelo econométrico}

Utilizando los parámetros del modelo econométrico y los datos de la ENAHO 2014 en lugar de los registros oficiales, es posible realizar una simulación del mecanismo de focalización: utilizando los datos reportados por los beneficiarios de los programas incluidos en la Encuesta Nacional, se puede simular cuál habría sido el grupo en el que se asignarían dentro del SIPO. En este caso, se observa en la Tabla $\underline{3}$ un patrón diferente: una tasa de filtración (incluye los vulnerables y no prioritarios) de $28,8 \%$ para Avancemos y $20,9 \%$ para Bienestar Familiar.

Estos indicadores muestran niveles más bajos en relación con la filtración que se observó utilizando el método de línea de pobreza, pero difieren de la información reportada en las bases de datos del IMAS. Existen consideraciones importantes que deben ser analizados en detalle incluidas en la siguiente sección, las cuales sugieren que usar exclusivamente el modelo econométrico actual (SIPO-2005) podría no ser el mejor camino para focalizar los programas sociales.

Tabla 3.

Distribución de los beneficiarios por grupo SIPO con datos de la ENAHO 2014

\begin{tabular}{lrrrrr}
\hline & Prioridad 1 & \multicolumn{1}{c}{ Prioridad 2 } & Vulnerable & No prioritario & Total \\
\hline Avancemos & 25.5 & 45.7 & 17.4 & 11.4 & 100.0 \\
Bienestar Familiar & 45.2 & 33.9 & 14.9 & 6.0 & 100.0 \\
\hline
\end{tabular}

Fuente: elaboración propia con base en datos de INEC (2014).

\section{Mejoramiento del mecanismo de focalización de los programas sociales}

El objetivo final de los programas sociales analizados en este trabajo es reducir la pobreza ofreciendo transferencias de corto plazo, ya sea mediante un subsidio temporal para cubrir necesidades básicas (Bienestar Familiar) o el desarrollo de competencias de las personas por medio de la condicionalidad que introduce el programa Avancemos. Para lograr este objetivo, la asignación de los beneficios debe ser lo más eficiente posible: llegar a aquellos que realmente necesitan la protección del Estado para utilizar los limitados recursos disponibles. Esta asignación eficiente implica diferentes dimensiones: el diseño del proceso de solicitud, el mecanismo para clasificar los hogares, las capacidades administrativas de las instituciones y las decisiones políticas que dan forma a los programas sociales. A pesar de que el análisis se centra en el mecanismo para clasificar los hogares, también se consideran en el análisis las otras dimensiones.

\subsection{Alcanzar a la población más pobre}

El primer paso para incluir a las personas pobres en un programa social focalizado es generalmente recoger cierta información acerca de sus niveles de vida e ingresos. Sin embargo, 12

Francisco Delgado Jiménez

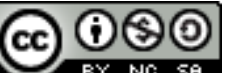

Revista Economía y Sociedad by Universidad Nacional is licensed under a CreativeCommons Reconocimiento-NoComercial- 
los canales por los cuales los candidatos solicitan un beneficio también pueden alterar el proceso de selección. Existen diferentes mecanismos: las personas pueden visitar una oficina del gobierno para pedir ayuda o los entes públicos pueden decidir cubrir un área geográfica en particular o un grupo específico y llegar a ellos.

Recientemente, se han añadido instrumentos importantes, como los mapas sociales, al stock de herramientas que el Estado costarricense puede utilizar para identificar a los hogares pobres, conocidos como de "barrido". Sin embargo, para aquellos programas gestionados por el IMAS, la auto-selección sigue siendo la forma más común de entrar en estos, lo cual significa que todos los interesados en un programa en particular deben mostrar su voluntad de iniciar una evaluación formal in situ para estudiar su vivienda y las condiciones socioeconómicas.

Entre los hogares pobres, los bajos niveles de educación y el acceso restringido a las instituciones formales son características comunes. En una situación en la que las instituciones gubernamentales no tienen un papel proactivo en busca aquellos hogares que necesitan protección social; el diseño del programa debe tener en cuenta cuáles son los mejores mecanismos para proporcionar información o para construir redes que permitan alcanzar y atraer a aquellos que realmente necesitan asistencia. En enero de 2015, en el marco de este trabajo, se llevó a cabo una pequeña encuesta ${ }^{8}$ de hogares en condiciones de vulnerabilidad para recopilar información valiosa sobre las oportunidades y desafíos que tienen para acceder a los programas sociales que se ofrecen y de pensar en los canales más adecuados para difundir información (medios de comunicación, redes, etc.). Los principales resultados de esta encuesta se describen en esta sección, y los datos se encuentran en los anexos $\underline{1}$ y $\underline{2}$.

Los resultados proporcionan pistas interesantes sobre la dinámica dentro de las comunidades, en cuanto a redes informales de apoyo y comunicación y sobre la tarea del gobierno para llegar a los hogares pobres. Actualmente, el gobierno se basa en la auto-identificación de los hogares para inscribirlos en los programas sociales, pero parece que muchos de ellos no tienen conocimiento de los beneficios de estos programas o si son elegibles. Esto tiene también un papel relevante para la determinación de quién es, al final, beneficiario de un programa social.

El mecanismo de auto-selección que se encuentra actualmente vigente para solicitar los beneficios de los programas sociales se explica por la limitada asignación de recurso humano, creando un obstáculo para el establecimiento de procesos de difusión a gran escala. Esta es una barrera clave especialmente para la población más desfavorecida, que es también la que tiene menos acceso a la información. La estrategia "Puente al Desarrollo" y la utilización de mapas

\footnotetext{
${ }^{8}$ La encuesta incluyó 25 jefas y jefes de hogar en el Barrio Los Ángeles, una comunidad urbana en San José que en la actualidad es atendida por la organización no gubernamental Fundación Techo. La comunidad es un asentamiento en condiciones de pobreza extrema localizado en Patarrá de Desamparados. Todos los participantes en la encuesta viven en situación de pobreza o extrema pobreza, si esa condición es aproximada por el método de línea de pobreza.
} 
sociales reconocen, en parte, este problema y suponen un abordaje de la atención social mucho más cercano a las familias en condición de pobreza. También sería recomendable el trabajo conjunto con otras organizaciones no gubernamentales que ya están en el campo, como un mecanismo informal para llegar a la población objetivo de los programas.

\subsection{Clasificación de los hogares solicitantes}

El modelo econométrico utilizado en el sistema de puntaje se basa en la Encuesta de Hogares de Propósitos Múltiples 2004 (Pacheco, Ramírez y Villegas, 2006). En 2010, el IMAS contrató a un equipo de consultores para revisar el modelo econométrico usando la Encuesta de Hogares de 2009, pero el algoritmo no fue implementado. Por otra parte, hubo cambios metodológicos para medir la pobreza que se introdujeron en la Encuesta a partir del año 2010. Esto explica por qué es necesaria una actualización para focalizar mejor a los pobres: algunas de las variables que se utilizan para evaluar sus condiciones de vida ya no son relevantes y sería recomendable la inclusión de algunas otras variables.

Una de las principales limitaciones y errores conceptuales que tiene el SIPO es que utiliza un modelo econométrico con parámetros estimados para los hogares de la Encuesta Nacional, pero los aplica a una agrupación distinta: las familias. Es decir, la unidad de análisis entre la encuesta y el modelo de atención actual del IMAS es distinta, lo que genera incentivos y sesgos importantes, como lo señalan Trejos y Sáenz (2007).

Uno de los parámetros clave del modelo es el llamado "percentil promedio". Esto representa un promedio simple del percentil de ingreso per cápita de las familias que comparten una característica común. Por ejemplo, es posible estimar el percentil promedio de las familias que tienen electricidad en la vivienda y un promedio para aquellos que no tienen. Esos parámetros fueron fijados con base en los datos de la EHPM 2004; por lo tanto, si una familia muestra cierta característica se le asigna dicho parámetro. Esta primera simulación permite afirmar lo siguiente:

- La mayoría de los percentiles promedio muestran niveles más bajos en 2014, lo que significa que durante los últimos 10 años ha habido una mejora en las condiciones de vida de los hogares ${ }^{9}$, a pesar de la mayor desigualdad en el ingreso observada en una mayor dispersión de los puntajes que obtienen las familias.

- Ha habido pocas mejoras en la reducción de las disparidades entre las distintas zonas. Para casi todas las variables y sus categorías, la zona rural muestra percentiles promedio más bajos en comparación con el área urbana.

\footnotetext{
${ }^{9}$ Por ejemplo, considere el caso de la variable "Techo". Si una familia pobre (bajo percentil) es capaz de mejorar su casa, esto significa que la categoría "sin techo" ahora contiene hogares mucho más pobres (por tanto, un percentil promedio más bajo), y la categoría "con techo" ahora incluye aquellos hogares pobres que lograron mejorar su casa (generando un percentil promedio también menor).
}

14

Francisco Delgado Jiménez

(c) (i) (2)

Revista Economía y Sociedad by Universidad Nacional is licensed under a CreativeCommons Reconocimiento-NoComercial- 
- Para algunas variables, la brecha en el percentil promedio entre las familias que muestran y no muestran algunas características no es tan amplia como en 2004. El caso más evidente es la variable "Teléfono celular", donde la diferencia entre categorías es menor tanto en zonas urbanas como rurales. Este resultado lleva a considerar si las variables con este comportamiento son relevantes o no en el escenario actual, especialmente para diferenciar a los hogares con menores ingresos.

- El factor llamado "Patrimonio" incluye una lista de diferentes aparatos electrónicos. Sin embargo, la nueva Encuesta Nacional de Hogares no indaga si un hogar posee lavadora, horno microondas, ducha de agua caliente o VHS; por lo tanto, en una nueva definición del modelo, estas variables deben ser excluidas debido a que la encuesta no permite estimar los percentiles promedio de estas características ${ }^{10}$.

Como se explicó antes, fue posible simular lo que hubiera sido el puntaje de todas las familias en la ENAHO 2014, con especial énfasis en los beneficiarios de los programas y en obtener medidas más robustas de filtraciones y subcobertura. Siguiendo con la simulación del modelo econométrico, en esta sección se analiza el desempeño del mecanismo de selección utilizando la muestra completa y no solo los beneficiarios.
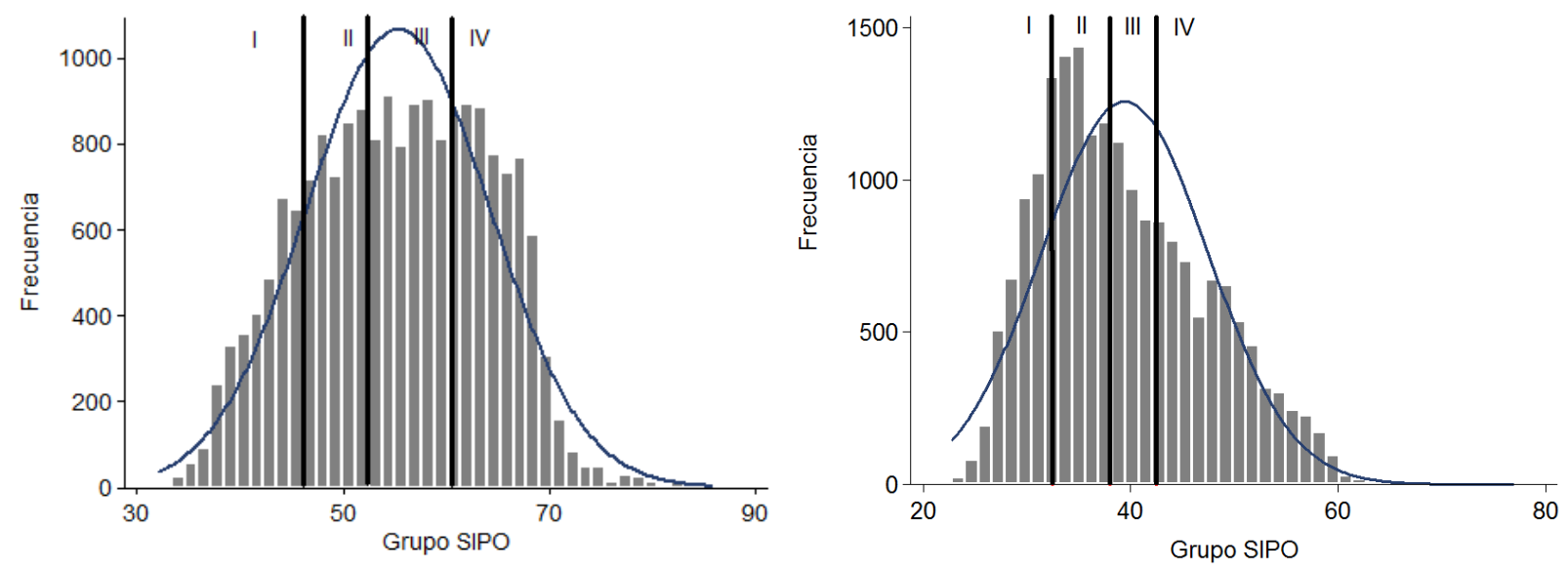

Figura 5. Simulación del puntaje (ENAHO 2014), distribución observada. Área urbana (izquierda) y rural (derecha). Fuente: estimación propia con base en INEC (2014).

Recordemos que las familias se dividen en cuatro grupos de acuerdo con su "puntaje SIPO". Los valores de corte entre los grupos (líneas verticales más sólidas en la Figura $\underline{5}$ ) también son parámetros del modelo. Los gráficos de la Figura $\underline{5}$ muestran información interesante y resume un hecho acerca de la pobreza en el país: la concentración y la gravedad de la pobreza en el

\footnotetext{
${ }^{10}$ En el ejercicio de simulación no fue posible estimar los percentiles promedio para estas variables. Con el fin de reproducir el modelo SIPO-2005, fue necesario calcular una puntuación para cada familia dentro de un intervalo: SIPO_min. en caso de una familia no posea ninguno de esos aparatos y SIPO_max. si tenían todos los electrodomésticos de los que no existe información. Para simplificar el análisis, se imputó un promedio de ambos.
} 
área rural. La distribución de las puntuaciones SIPO es ahora más dispersa en las zonas urbanas y rurales, en comparación con el análisis de 2004. Pero más importante, se puede observar que casi a la mitad de la población se le daría un rango de prioridad cuando se trata de asistencia social; un resultado que es evidentemente inconsistente con todas las mediciones de pobreza y las descripciones de la realidad socioeconómica del país. Esta contradicción es también una sugerencia para una actualización del modelo en términos de a) los valores de corte utilizados para determinar la composición de los diferentes grupos, o b) el número de grupos utilizados para caracterizar los hogares.

También es interesante conocer la composición de los diferentes grupos, no solo sus frecuencias. El siguiente paso es analizar las condiciones socioeconómicas de los hogares de cada grupo, dando énfasis a la situación de pobreza.

Es posible observar que el mecanismo de selección es efectivo para reducir el error de exclusión, en tanto los hogares en pobreza y pobreza extrema son ubicados en los grupos 1 y 2 casi totalmente. Sin embargo, el modelo econométrico no consigue reducir el error de inclusión. En particular, se observa en la Figura $\underline{6}$ una importante proporción de hogares no pobres en el segundo grupo de prioridad.

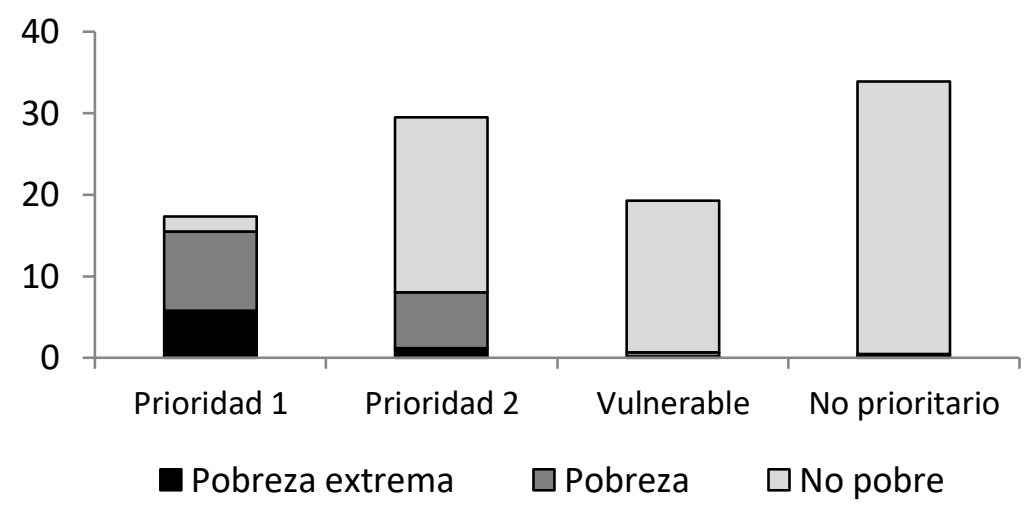

Figura 6. Distribución de la población del país por grupo SIPO y nivel de pobreza. Fuente: elaboración propia con datos de INEC (2014).

\section{Actualización del mecanismo de puntaje}

La estructura y el diseño de puntaje actual hacen que ese mecanismo constituya un buen método para identificar un problema tan complejo como el de la pobreza, pero requiere de ajustes. El proceso de actualización que se sugiere aquí está dirigido a mejorar el desempeño de la focalización de ese instrumento, con el fin de reducir tanto los errores de exclusión como de inclusión.

16

Francisco Delgado Jiménez

(c) (†) 8 ()

Revista Economía y Sociedad by Universidad Nacional is licensed under a CreativeCommons Reconocimiento-NoComercial- 
En primer lugar, debido a las inconsistencias que genera tener un modelo econométrico basado en hogares aplicado a una población agrupada en familias, resulta conveniente que el IMAS migre hacia el hogar como unidad de análisis. Con eso se daría fin a una sobreestimación de la población objetivo, y se daría un abordaje de la pobreza tomando en consideración redes de apoyo que van más allá de los vínculos de consanguinidad.

La revisión del mecanismo de puntaje también puede dar lugar a un nuevo modelo con variables adicionales que proporcionen más información para determinar mejor la elegibilidad para un programa social. Si ese es el caso, es relevante tener en cuenta que la Ficha de Información Social debe ser actualizada para incluir esa nueva información cuando los trabajadores sociales realicen la evaluación in situ. Con base en estudios e informes previos que exploran el fenómeno de la pobreza en el país (por ejemplo, Trejos y Sáenz, 2007 o Sauma y Trejos, 2014) y también la experiencia internacional (Ministerio de Desarrollo Social, 2010), sería conveniente incluir variables como las siguientes:

- Presencia de miembros con discapacidad en el hogar. Los datos sugieren que existen diferencias, en promedio, entre aquellos hogares en los que al menos uno de sus miembros tiene una discapacidad, tanto en zonas urbanas como rurales.

- Mujeres jefas de hogar. La brecha de género en los ingresos es un problema social que se ha estudiado en gran medida y los datos también apoyan la idea de que las mujeres a cargo de sus familias tienen más probabilidades de ser pobres.

- Inestabilidad laboral. Hay varias condiciones que pueden explicar las constantes fluctuaciones en el ingreso per cápita de las familias, un fenómeno que tiene un impacto negativo en la satisfacción de las necesidades básicas. Por ejemplo, cuando existe incertidumbre sobre los ingresos futuros, las familias podrían posponer la decisión de mejorar sus viviendas, asistir a la escuela o visitar a un médico. Especialmente para los trabajadores no calificados o los del sector informal, la inestabilidad laboral, la estacionalidad y otros shocks económicos son preocupaciones importantes y el método de focalización debe tener en cuenta estas situaciones.

Asimismo, la definición de las cuatro categorías (grupos SIPO) en el modelo econométrico actual resulta problemático, sobre todo porque no permite diferenciar a familias con condiciones muy heterogéneas: el Grupo 2 es el más diverso y el que permite la inclusión de familias no pobres. Sería conveniente hacer agrupaciones más pequeñas.

Por último, la utilización de registros administrativos para verificar información autodeclarada puede ser una herramienta fundamental para reducir las filtraciones. En línea con lo anterior, una nueva plataforma denominada Sistema Nacional de Información y Registro Único de Beneficiarios del Estado (SINIRUBE) fue establecida recientemente por ley, para finalmente 
crear un registro unificado de beneficiarios de todos los programas sociales financiados por el Estado. Este sistema estará bajo la responsabilidad del IMAS, lo que agrega otra herramienta para promover la coordinación entre otros entes públicos. Mediante consultas a registros administrativos sobre ingresos y patrimonio de los hogares (como las cotizaciones a la seguridad social o las propiedades registradas), también será posible realizar un control cruzado en la asignación de los beneficios.

\section{Consideraciones finales}

Los hallazgos más sobresalientes de este análisis se relacionan con la forma en que se mide la pobreza y los mecanismos mediante los cuales los programas alcanzan a la población objetivo. Específicamente: (1) el modelo econométrico utilizado para clasificar a las familias presenta inconsistencias que sobreestiman la pobreza total y (2) una encuesta original revela que existe conocimiento acerca de los programas, pero los solicitantes potenciales carecen de información sobre los beneficios o las condiciones de elegibilidad.

Datos de la Encuesta Nacional de Hogares de 2014 revela que alrededor del 25\% de los estudiantes que recibieron becas Avancemos pertenecía a hogares que no eran pobres ni vulnerables y alrededor del $60 \%$ de los estudiantes pobres y de bajos ingresos no recibió el beneficio. En el caso de Bienestar Familiar, el IMAS ha tenido dificultad para aumentar la cobertura más allá del $10 \%$ de la población objetivo y casi el $40 \%$ de los beneficios se asignan a hogares no pobres.

Con el fin de tener un efecto más decisivo en la reducción de la pobreza a través de los programas selectivos existentes, este diagnóstico sugiere que el IMAS debe seguir una estrategia que aborde varios frentes: (1) acercarse a la población más pobre para mejorar la focalización y difundir información sobre los programas a través de redes informales y ampliar la cobertura de atención de las personas cogestoras sociales, (2) hacer una actualización del modelo econométrico con el propósito de mejorar la focalización, y (3) hacer control cruzado de información con registros administrativos mediante el SINIRUBE para reducir las filtraciones.

\section{Referencias}

Asamblea Legislativa (1970). Expediente No. 4334. Proyecto de ley de creación del Instituto Mixto de Ayuda Social. San José, Costa Rica. Recuperado de: http://expedientes.asamblea.go.cr/elntegrator31/document.aspx?doc $=5793$

Coady, D., Grosh, M., \& Hoddinott, J. (2004). Targeting of Transfers in Developing Countries: Review of Lessons and Experience. doi: https://doi.org/10.1596/0-8213-5769-7 
Contraloría General de la República (2006). Identificación y caracterización de los programas de asistencia y promoción social y algunas posibilidades de mejorar su integración (Informe DFOE-SO-30-2006.) Recuperado de: http://cgrfiles.cgr.go.cr/publico/docs cgr/2006/SIGYD_D_2006018313.doc

Instituto Nacional de Estadística y Censos (2014). Encuesta Nacional de Hogares, Julio 2014. Archivos de datos. Recuperado de: http://sistemas.inec.cr/pad4/index.php/catalog/148/study-description

Instituto Mixto de Ayuda Social (2009). Manual único para el otorgamiento de beneficios institucionales. Recuperado de https://www.imas.go.cr/ayuda social/ibs/IBS\%20\%20Manual\%20aspectos\%20IBS.pdf

Instituto Mixto de Ayuda Social (2014). Directriz de priorización de atención de la pobreza. Recuperado

de: http://www.imas.go.cr/acerca imas/normativa/Directrices/Directriz de priorizacion de atencion de la pobreza - Presidencia Ejecutiva IMAS.pdf

Ministerio de Desarrollo Social (2010). Comité de Expertos Ficha de protección social (Informe final). Recuperado de: http://www.ministeriodesarrollosocial.gob.cl/btca/txtcompleto/mideplan/c.e-fpsinfinal.pdf

Pacheco, C., Ramírez, F., \& Villegas, G. (2006). Informe: Actualización y desarrollo del modelo estadístico para el cálculo del puntaje. Construcción del modelo SIPO-2005. San José: Instituto Mixto de Ayuda Social.

Sauma, P., \& Trejos, J. D. (2014). Reducir la pobreza en Costa Rica es posible: Propuestas para la acción. Serie de Cuadernos de Desarrollo Humano 1-2014. Recuperado de: http://www.cr.undp.org/content/dam/costa_rica/docs/undp_cr_reducirpobreza_20 14.pdf

Solís, P. (2012). Instituto Mixto de Ayuda Social: 40 años de historia (1971-2011). San Salvador, El Salvador.

Trejos, J. D., \& Sáenz, M. I. (2007). La selección de los beneficiarios para los programas contra la pobreza: La experiencia del IMAS. Revista Economía y Sociedad, 12 (31-32), 79-100. Recuperado de: http://www.revistas.una.ac.cr/index.php/economia/article/view/175/134 
Víquez, R. (2005). Sistema de Identificación de la Población Objetivo: SIPO en Costa Rica. (Serie de Documentos de Discusión sobre la Protección Social, No. 530). Recuperado de: http://documentos.bancomundial.org/curated/es/694471468027536311/pdf/32760 Orev.pdf

\section{Anexos}

\section{Anexo 1. Acerca del modelo econométrico SIPO del IMAS}

El modelo econométrico utilizado por el IMAS como "proxy means test", denominado también Puntaje SIPO, consiste en utilizar variables observables, asignarles una ponderación y construir un indicador que permita priorizar a los hogares que podrían ser sujetos de la intervención. El modelo consta de 28 variables que se agrupan en 6 factores, como se detalla a continuación:

Tabla A.1.

Factores, subfactores y variables que componen el Modelo SIPO-2005

\begin{tabular}{|c|c|c|}
\hline FACTOR & SUBFACTOR & VARIABLE \\
\hline \multirow{10}{*}{ VIVIENDA } & \multirow{3}{*}{ PROTECCIÓN AMBIENTAL } & MATERIAL DE PAREDES \\
\hline & & MATERIAL DEL PISO \\
\hline & & TECHO CON O SIN CIELO RASO \\
\hline & HACINAMIENTO & $\begin{array}{l}\text { TOTAL DE PERSONAS EN LA VIVIENDA/TOTAL DE CUARTOS PARA DORMIR } \\
\text { EN LA VIVIENDA }\end{array}$ \\
\hline & \multirow{6}{*}{ SANEAMIENTO Y CONFORT } & ABASTECIMIENTO DE AGUA \\
\hline & & SISTEMA ELIMINACIÓN EXCRETAS \\
\hline & & DISPONIBILIDAD BAÑO \\
\hline & & SUMINISTRO ELÉCTRICO \\
\hline & & FUENTE DE ENERGÍA PARA COCINAR \\
\hline & & MEDIO DE ELIMINACIÓN DE BASURA \\
\hline \multirow[b]{2}{*}{ EDUCACIÓN } & ESCOLARIDAD DEL JEFE & NIVEL EDUCATIVO DEL JEFE \\
\hline & $\begin{array}{l}\text { ESCOLARIDAD DE OTROS } \\
\text { MIEMBROS }\end{array}$ & $\begin{array}{l}\text { NIVEL EDUCATIVO DE OTROS MIEMBROS DEL HOGAR CON EDAD DE } 18 \\
\text { AÑOS O MÁS }\end{array}$ \\
\hline \multirow[b]{2}{*}{ OCUPACIÓN } & OCUPACIÓN DEL JEFE & CONDICIÓN DE ACTIVIDAD Y CATEGORÍA OCUPACIONAL DEL JEFE \\
\hline & $\begin{array}{l}\text { OCUPACIÓN DE OTROS } \\
\text { MIEMBROS }\end{array}$ & $\begin{array}{l}\text { GRUPO DE OCUPACIÓN DE OTROS MIEMBROS DEL HOGAR CON EDAD DE } \\
18 \text { AÑOS O MÁS }\end{array}$ \\
\hline \multirow{7}{*}{ PATRIMONIO } & VIVIENDA & TENENCIA DE LA VIVIENDA \\
\hline & \multirow{4}{*}{ EQUIPAMIENTO 1} & POSESIÓN DE TV A COLOR \\
\hline & & POSESIÓN DE REFRIGERADORA \\
\hline & & POSESIÓN DE LAVADORA \\
\hline & & POSESIÓN DE TELÉFONO RESIDENCIAL \\
\hline & \multirow{2}{*}{ EQUIPAMIENTO 2} & POSESIÓN DE VHS \\
\hline & & POSESIÓN DE TELÉFONO CELULAR \\
\hline
\end{tabular}

20

Francisco Delgado Jiménez

(c) (†) 8 ()

Revista Economía y Sociedad by Universidad Nacional is licensed under a CreativeCommons Reconocimiento-NoComercial- 


\begin{tabular}{|l|l|l|}
\hline \multicolumn{1}{|c|}{ FACTOR } & SUBFACTOR & \multicolumn{1}{c|}{ VARIABLE } \\
\hline \multirow{5}{*}{} & & POSESIÓN DE DUCHA AGUA CALIENTE \\
\cline { 3 - 3 } & POSESIÓN DE TANQUE PARA ALMACENAR AGUA \\
\cline { 3 - 3 } & POSESIÓN DE COMPUTADORA \\
\cline { 2 - 3 } & POSESIÓN DE VEHÍCULO \\
\cline { 2 - 3 } & POSESIÓN DE HORNO MICROONDAS \\
\hline ASEGURAMIENTO & CATEGORIA DE ASEGURAMIENTO DE LOS MIEMBROS DEL HOGAR \\
\hline INGRESO & INGRESO PER-CÁPITA DEL HOGAR \\
\hline
\end{tabular}

Fuente: Pacheco, C., Ramírez, F., \& Villegas, G. (2006).

El modelo se compone de variables tanto numéricas como categóricas. Por ejemplo, el ingreso per cápita del hogar es una variable numérica; sin embargo, la variable material del piso es categórica, ya que las opciones de respuesta son un conjunto de materiales y no un número.

Para poder asignar a cada categoría de una variable un valor cuantitativo (escala métrica) se utiliza el percentil promedio. Esto busca que puntuar el grado con que la categoría expresa una condición socioeconómica más o menos precaria; es decir, se pasa de una escala cualitativa a otra cuantitativa. "Como criterio para calificar el grado en que una determinada categoría de una variable expresa una peor o mejor condición socioeconómica de un hogar, se utilizó el percentil promedio del ingreso per-cápita de los hogares que se ubican en dicha categoría. En general, en las categorías que se establecen, se observan diferencias importantes en sus respectivos percentiles" (Pacheco, Ramírez, \& Villegas, 2006, p.14).

Una vez que cada variable y sus categorías tienen un percentil asociado, se hace una ponderación de las variables, los subfactores y factores, por medio de la técnica estadística multivariada denominada análisis de componentes principales (ACP). En este caso, los factores, subfactores y variables que se correlacionan más con la pobreza, tendrán coeficientes o ponderaciones mayores.

\section{Anexo 2. Aspectos sobre la encuesta aplicada a una población en condición de pobreza}

La encuesta incluyó 25 jefes de hogar en el Barrio Los Ángeles, una comunidad urbana en San José ${ }^{11}$ que en la actualidad es atendida por la organización no gubernamental Fundación Techo. Todos los participantes en la encuesta viven en situación de pobreza o extrema pobreza, si esa condición es aproximada por el método de línea de pobreza.

Uno de los hallazgos más sobresalientes de esta encuesta es el hecho de que muchos de los participantes tenían conocimiento de la existencia de los dos programas sociales (Avancemos y

11 La comunidad está localizada en Patarrá de Desamparados y es caracterizada como un asentamiento en condiciones de pobreza extrema, según Fundación Techo (2014). 
Bienestar Familiar), pero la mayoría de ellos tuvo dificultades para identificar los beneficios proporcionados por cada uno de ellos. Entre los que han oído hablar de Avancemos, una parte de ellos (25\%) habían recibido información en la escuela de sus hijos; que uno podría pensar que es el mecanismo más directo y efectivo. Un tercio de los participantes que sabían de Avancemos obtuvieron información a través de familiares y vecinos, lo cual destaca la importancia de las redes informales. La mayoría (42\%) se enteró a través de los medios de comunicación, especialmente en televisión, ya que el programa era bastante publicitado hace unos años. En el caso de los participantes que sabían de Bienestar Familiar, los resultados cambian ligeramente. Menos personas habían oído hablar de él en los medios de comunicación (14\%) y el papel de las redes informales fue más fuerte (50\%).

Cuando se les preguntó dónde o cómo buscarían más información en caso de que estuvieran interesados en aplicar a alguno de estos programas, un grupo importante de ellos (40\%) reconoció que el IMAS es la agencia que administra los programas y la mayoría de ellos también sabía acerca de la ubicación de la oficina regional más cercana. Escuela, medios de comunicación o los vecinos fueron mencionados por el $20 \%$, y fue interesante oír de un $15 \%$ de los encuestados que buscaría en los sitios web de las instituciones para obtener información. Alrededor del $25 \%$ de ellos no sabría adónde ir.

A pesar de que estos resultados no permiten la inferencia estadística, proporcionan pistas interesantes sobre la dinámica dentro de las comunidades y sobre la tarea del gobierno de llegar a las familias pobres. Actualmente, el gobierno se basa en la auto-identificación de las familias para inscribirlos en los programas sociales, pero parece que muchos de ellos no tienen conocimiento de los beneficios de estos programas o si son elegibles. Esto tiene también un papel relevante la determinación de quién es, al final, beneficiario de un programa social. Por otra parte, los que estaban relativamente más conscientes de los programas tenían acceso a la información a través de diferentes canales de comunicación y también de familiares y vecinos. Este hallazgo ofrece una guía a los entes públicos que no han desarrollado una estrategia o la capacidad institucional para llegar a las familias pobres.

22 
Economía y Sociedad, Vol. 22, № 52, julio-diciembre 2017, pp. 1-24

EISSN: 2215-3403 URL http://www.revistas.una.ac.cr/economia

\section{Cuestionario para la realización de la encuesta}

Encuesta Programas Sociales

Distrito: Patarrá Cantón: Desamparados Provincia: San José

\section{Características del hogar}

1.Número de miembros en el hogar:

2.Número de niños:

3.Número de adultos mayores de 65:

4. Esta casa es...

$\square$ Alquilada $\square$ Propia $\square$ Otro
5.¿El jefe de hogar es mujer? $\square$ Sí $\square$ No

6. ¿Cuál es la ocupación del o la jefe de hogar?

7. Cuántos niños/jóvenes asisten al kinder, escuela, colegio o universidad?

\section{Acerca de los programas sociales}

A8.¿Ha escuchado del programa "Avancemos"? $\square$ Sí (Ir a la pregunta A9) $\square$ No (Ir a la pregunta B8)

A9. ¿Cómo supo de ese programa?

$\square$ Familiar

$\square$ Iglesia

$\square$ Vecinos/Amigos

$\square$ Prensa

$\square$ Escuela/Colegio

$\square$ Trabajador social

$\square$ Otro:
A10. ¿Sabe cuál institución está A11. ¿Sabe cuáles son los a cargo de ese programa? beneficios del programa?

$\square$ Sí. ¿Cuál?

$\square$ No $\quad \square$ No
A12. Es beneficiario del programa Avancemos?

A13. ¿Qué le dieran la beca fue...?

$\square$ Muy fácil (Ir a la B8)

$\square$ Fácil (Ir a la B8)

$\square$ Difícil (Ir a la A13a)

$\square$ Muy difícil (Ir a la A13a) $\square$ Sí (Ir a la pregunta A13 ) $\square$ No (Ir a la pregunta A14)

A13a. Si no fue fácil, ¿qué obstáculos o complicaciones tuvo?

(Ir a la B8)

A14. ¿Alguna aplicó a una beca Avancemos? $\square$ Sí , pero no fue seleccionado (Ir a la B8 ) $\square$ No (Ir a la A15)

A15. ¿Por qué no aplicó? 
B8.¿Ha escuchado de las ayudas del IMAS? $\square$ Sí (Ir a la pregunta B9) $\square$ No (Ir a la pregunta 16)

B9. ¿Cómo supo de esas ayudas?

B11. ¿Sabe qué tipo de ayuda brinda el IMAS?

$\square$ Familiar $\quad \square$ Iglesia

$\square$ Vecinos/Amigos $\quad \square$ Prensa

$\square$ Sí ¿Cuáles?

$\square$ Escuela/Colegio $\square$ Trabajador social

$\square$ No

$\square$ Otro:

B12. ¿Es beneficiario de otras ayudas del IMAS?

$\square$ Sí (Ir a la pregunta B13 ) $\square$ No (Ir a la pregunta B14)

B13. Que le dieran esa beca fue...?

B13a. Si no fue fácil, ¿̇ué obstáculos o complicaciones

$\square$ Muy fácil (Ir a la B8) tuvo?

$\square$ Fácil (Ir a la B8)

$\square$ Difícil (Ir a la A13a)

$\square$ Muy difícil (Ir a la A13a)

(Ir a la 16)

B14. ¿Alguna vez aplicó a las ayudas del IMAS? $\square$ Sí , pero no fue seleccionado (Ir a la 16) $\square$ No (Ir a la B15)

B15. ¿Por qué no aplicó?

16. ¿Si estuviera interesado en aplicar a algún programa financiado por el Estado, dónde buscaría más información?

17. ¿Cuál es el ingreso total del hogar...?

$\begin{array}{ll}\square \text { Menos de } 350.000 \text { colones } & \square \text { Entre } 990.000 \text { y } 1.700 .000 \\ \square \text { Entre } 350.000 \text { y } 550.000 \text { colones } & \square \text { Más de } 1.700 .000 \\ \square \text { Entre } 550.000 \text { y } 990.000 & \square \text { NS/NR }\end{array}$

Fuente: elaboración propia.

24

Francisco Delgado Jiménez

(c) (1) 8 (2)

Revista Economía y Sociedad by Universidad Nacional is licensed under a CreativeCommons Reconocimiento-NoComercial- 